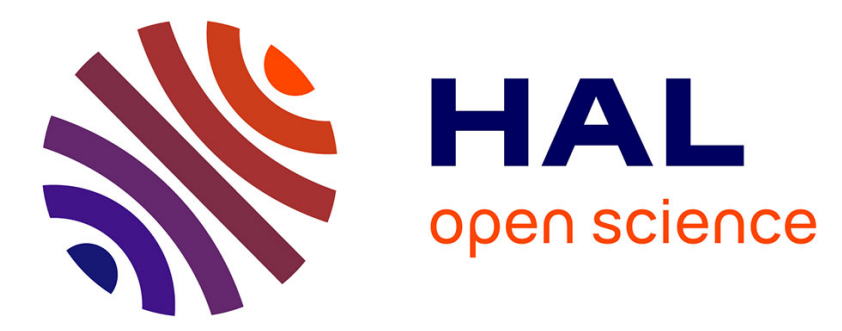

\title{
On nonlinear distributed parameter model predictive control strategy: On-line calculation time reduction and application to an experimental drying process
}

\author{
Pascal Dufour, Youssoufi Touré, Denise Blanc, Pierre Laurent
}

\section{- To cite this version:}

Pascal Dufour, Youssoufi Touré, Denise Blanc, Pierre Laurent. On nonlinear distributed parameter model predictive control strategy: On-line calculation time reduction and application to an experimental drying process. Computers \& Chemical Engineering, 2003, 27 (11), pp.1533-1542. 10.1016/S00981354(03)00099-1 . hal-00352371v2

\section{HAL Id: hal-00352371 \\ https://hal.science/hal-00352371v2}

Submitted on 21 Jan 2009

HAL is a multi-disciplinary open access archive for the deposit and dissemination of scientific research documents, whether they are published or not. The documents may come from teaching and research institutions in France or abroad, or from public or private research centers.
L'archive ouverte pluridisciplinaire HAL, est destinée au dépôt et à la diffusion de documents scientifiques de niveau recherche, publiés ou non, émanant des établissements d'enseignement et de recherche français ou étrangers, des laboratoires publics ou privés. 
This document must be cited according to its final version which is published in a journal as:

\author{
P. Dufour ${ }^{1}$, Y. Touré ${ }^{2}$, D. Blanc ${ }^{3}$, P. Laurent ${ }^{1}$ \\ "On nonlinear distributed parameter model predictive control strategy: \\ On-line calculation time reduction \\ and application to an experimental drying process", \\ Computers and Chemical Engineering, ISSN: 0098-1354 \\ 27(11), pp. 1533-1542, 2003. \\ http://dx.doi.org/10.1016/S0098-1354(03)00099-1
}

All open archive documents of Pascal Dufour are available at: http://hal.archives-ouvertes.fr/DUFOUR-PASCAL-C-3926-2008

The professional web page ( $\mathrm{Fr} / \mathrm{En}$ ) of Pascal Dufour is: http://www.lagep.univ-lyon1.fr/signatures/dufour.pascal

1

Université de Lyon, Lyon, F-69003, France; Université Lyon 1;

CNRS UMR 5007 LAGEP (Laboratoire d'Automatique et de GEnie des Procédés),

43 bd du 11 novembre, 69100 Villeurbanne, France

Tel +33 (0) 472431845 - Fax +33 (0) 472431699

http://www-lagep.univ-lyon1.fr/ http://www.univ-lyon1.fr http://www.cnrs.fr

2

Université d'Orléans,

UPRES EA 2078 LVR (Laboratoire de Vision et de Robotique ),

63 Av de Lattre de Tassigny, 18020 Bourges Cedex, France

http://www.bourges.univ-orleans.fr/rech/lvr/

3

INSA de Lyon;

LAEPSI (Laboratoire d'Analyse Environnementale des Procedes et des Systemes Industriels);

Bâtiment Said Carnot, 9 rue de la Physique

69621 Villeurbanne cedex, France

http://laepsi.insa-lyon.fr/ 
On nonlinear distributed parameter model

\title{
predictive control strategy: on-line calculation \\ time reduction and application to an experimental drying process.
}

\author{
Dufour P. ${ }^{\mathrm{a}, 1}$, Touré Y. ${ }^{\mathrm{b}}$, Blanc D. ${ }^{\mathrm{c}}$ and Laurent P. ${ }^{\mathrm{a}}$ \\ ${ }^{\mathrm{a}}$ LAGEP, Université Claude Bernard Lyon 1, UMR CNRS 5007 \\ 43, Bd du 11 Novembre 1918, 69622 Villeurbanne Cedex, France. \\ ${ }^{\mathrm{b}}$ LVR, Université d'Orléans, UPRES EA 2078 \\ 63, Av de Lattre de Tassigny, 18020 Bourges Cedex, France. \\ ${ }^{\mathrm{c}}$ LAEPSI, INSA Lyon
}

Bat. Sadi Carnot, 9 rue de la physique, 69621 Villeurbanne Cedex, France.

\begin{abstract}
It is now recognized that model predictive control (MPC) is an interesting alternative for real-time control of industrial processes. In the meantime, some problems do still remain in progress: for theoretical aspects, the a priori guarantee of the stability and for the practical aspects, the guarantee of sufficient time to solve to optimization problem at each sampled time positions. In this paper, we propose a global method that aims to reduce the on-line calculation time due to the PDE
\end{abstract}


model based optimization task resolution. It is addressed for a particular class of systems not very often studied in this context: systems described by partial differential equations (PDEs) which are, in the present case, nonlinear and parabolic. In order to decrease the computational burden, the nonlinear PDE system is solved offline. Then, a linearized PDE model around the previous off-line behavior is used to find the optimal variations for the on-line predictive control. The real-time control application given is concerned with a infrared drying process of painting film.

Key words:

Model predictive control, nonlinear partial differential equations, internal model control, real-time control, drying process.

\section{Introduction}

The main objective of this paper is to extend MPC to systems modeled by nonlinear PDEs. Even with only one spatial dimension, these systems are not often treated, especially in the nonlinear case. One has to point out that each time that phenomena are taking place spatially and that one geometrical dimension as more impact than others do, the hypothesis of unidimensionality becomes realistic. Explicitly, transport reaction phenomena with significant diffusive and convective phenomena are typically characterized by severe nonlineari-

ties and spatial variations, and are naturally described by partial differential equations. Examples of such processes include tubular reactors, packed bed reactors, absorption columns, drying or curing processes. The large number of real applications that results in such PDE models constitutes the motivation $\overline{1}$ Corresponding author: email dufour@lagep.univ-lyon1.fr, phone (33) 4724318 78, fax (33) 472431699 
for this work.

In control theory, due to the complexity of the problem, relatively few studies are devoted to control of processes explicitly characterized by a PDE model. Various methods are proposed for control of such distributed parameter systems but there is no general framework yet. Recently, Christofides developed order reduction by partitioning the eigenspectrum of the operator of the PDE system [8,12] and methods based on approximate inertial manifold for spatial discretization of the PDE [7,2]. A comprehensive study of his work can be found in his book [9]. Other works for controller synthesis of nonlinear PDE systems are based on symmetry groups, infinitesimal generators and invariant conditions $[23,15]$. Concerning [16,31], finite dimensional controller are obtained through model reduction based on various methods: singular value decomposition, Karhunen-Loéve expansion or eigenfunction method. In [3], stability conditions for closed loop control of linear PDE with finite dimension controller are given in time domain and frequency domain through semigroup analysis.

The framework presented here address the boundary control of nonlinear parabolic PDEs systems characterized by complex nonlinearities in the spatial domain and at the boundary as well. Moreover, this strategy has to be able to be implementable for on-line control, taking into account of natural physical limitations or specifications (constraints) inherent to many processes. An advanced model based control strategy, developed for ordinary differential equation systems, is well dedicated to solve this constrained problem: model predictive control. MPC or receding horizon control refers to a class of control algorithms in which a dynamic process model is used to predict and optimize process performance. The idea is to solve, at each sample time, an open-loop optimization problem over a finite prediction horizon in order to find the value 
of the manipulated variable that has to be implemented. The procedure is reiterated at the next sample time with the update of process measurements. Today, MPC has become an advanced control strategy widely used in industry. Indeed, MPC is well suited for high performance control since constraints can be explicitly incorporated into the formulation of the control problem. Therefore, it is not a surprise to see that it is an important tool for control engineers where plant being controlled are sufficiently slow for its implementation: indeed, one has to be able to solve an on-line optimization problem. More details and references on MPC can be found in $[1,21,19,27,26]$. From a practical point of view, the drawback of MPC is the computational time aspect. Indeed, the model aims to predict the future dynamic behavior of the process output over a finite prediction horizon and has to be solved during the on-line constrained optimization problem resolution.

The contribution of this paper is to provide a MPC framework for such PDE systems to reduce the on-line resolution time at 3 levels, as applied in [10] for the control of a catalytic reverse flow reactor. First, the control structure is an adaptation of MPC with internal model control (IMC) structure where the nonlinear PDE system (solved off-line) and a linearized PDE system (solved during the on-line optimization task) are both used in order to decrease the computational burden. Among other things, use of IMC structure permits to use numerical resolution methods less accurate than methods in other MPC formulations. Secondly, the control strategy is concerned with on-line resolution that aims to correct off-line structure results. In the third part, for the class of constraints considered here, methods of transformation and penalization are proposed, hence allowing the use of optimization algorithm faster and less restricting than others. Finally, the last part of the paper is concerned with the experimental IMC-MPC for infrared drying process of painting film. 


\section{IMC-MPC formulation}

\subsection{Class of continuous time model considered}

The class of single input single output (SISO) one dimensional nonlinear parabolic PDEs with boundary control considered is:

$$
\left(\mathcal{S}_{N L}\right)\left\{\begin{array}{l}
\frac{\partial \underline{x}(z, t)}{\partial t}=F_{d}(\underline{x}(z, t)) \quad \forall z \in \Omega, t>0 \\
F_{b}(\underline{x}(z, t), u(t))=0 \quad \forall z \in \partial \Omega, t>0 \\
\underline{x}(z, 0)=\underline{x}_{i} \quad \forall z \in \Omega \cup \partial \Omega \\
y_{m}(t)=C \underline{x}(z, t) \quad \forall z \in \Omega \cup \partial \Omega, t>0
\end{array}\right.
$$

where $z$ is the independent space variable, $\Omega$ is the spatial domain and $\partial \Omega$ its boundary, $t$ is the independent time variable. $\underline{x}$ is the state vector in a Hilbert Space, $u$ is the control or manipulated variable (MV) in $\mathbb{R}, y_{m}$ is the model output in $\mathbb{R} . F_{d}$ and $F_{b}$ are nonlinear operator [4]. $C$ is a linear operator [24].

Assumption 1 The solution for $\left(\mathcal{S}_{N L}\right)$ is unique and depends continuously on the initial value.

Moreover, the control of process described by such model is simultaneously subject to:

- constraints on the magnitude and velocity of the MV: 


$$
\begin{cases}u_{\min } \leq u(t) \leq u_{\max } & \forall t>0 \\ \dot{u}_{\min } \leq \dot{u}(t) \leq \dot{u}_{\max } & \forall t>0\end{cases}
$$

- $n$ general process output constraints on the controlled variable (CV) $y_{p}$ related to operating conditions, safety, quality:

$$
g_{i}\left(y_{p}(t), u(t)\right) \leq 0 \quad \forall t>0, \forall i \in \mathcal{I}_{1}^{n}=\{1, \ldots, n\}
$$

Assumption 2 There exists $u(t)=u_{0}(t)$ leading to the particular representation $\left(\mathcal{S}_{0}\right)$ of $\left(\mathcal{S}_{N L}\right)$ described by the triplet $\left\{u(t)=u_{0}(t), \underline{x}(z, t)=\underline{x}_{0}(z, t), y_{m}(t)=\right.$ $\left.y_{0}(t)\right\}$

Variations around this triplet are given by:

$$
\left\{\begin{array}{l}
u(t)=u_{0}(t)+\Delta u(t) \\
\underline{x}(t)=\underline{x}_{o}(t)+\Delta \underline{x}(t) \\
y_{m}(t)=y_{0}(t)+\Delta y_{m}(t)
\end{array}\right.
$$

where sufficiently small variations are described by the time-varying linearized model $\left(\mathcal{S}_{T V L}\right)$ obtained about $\left(\mathcal{S}_{0}\right)$ :

$$
\left(\mathcal{S}_{T V L}\right)\left\{\begin{array}{l}
\frac{\partial \underline{x}(z, t)}{\partial t}=A_{d}(t) \Delta \underline{x}(z, t) \quad \forall z \in \Omega, t>0 \\
A_{b}^{x}(t) \Delta \underline{x}(z, t)+A_{b}^{u}(t) \Delta u(t)=0 \quad \forall z \in \partial \Omega, t>0 \\
\Delta \underline{x}(z, 0)=\underline{0} \quad \forall z \in \Omega \cup \partial \Omega \\
\Delta y_{m}(t)=C \Delta \underline{x}(z, t) \quad \forall z \in \Omega \cup \partial \Omega, t>0
\end{array}\right.
$$

where the time-varying linear operators $A_{d}(t), A_{b}^{x}(t)$ and $A_{b}^{u}(t)$ are obtained 
from the linearization of $\left(\mathcal{S}_{N L}\right)$ about the behavior described by $\left(\mathcal{S}_{0}\right)$ [14].

Assumption 3 The solution for $\left(\mathcal{S}_{T V L}\right)$ is unique and depends continuously on the initial value.

\subsection{Discrete time MPC formulation}

One of the advantage of MPC formulation as a constrained optimization problem is that a large number of control problems can be stated. It covers trajectory tracking for a $\mathrm{CV}$, minimization of any economic function, minimization of energy supply under technical specifications, etc [11]. Therefore, one can consider the following general task of minimizing, under some constraints, the cost function $J$ (also named performance index):

$$
\min _{\tilde{u}} J(\tilde{u})=\sum_{j} g\left(y_{r e f}(j), y_{p}(j), u(j-1)\right) \forall j \in \mathcal{J}_{1}^{N_{p}}=\left\{k+1, \ldots, k+N_{p}\right\}(6)
$$

where $k$ is the actual discrete time index, $j$ is the discrete time index, $N_{p}$ is the receding horizon and $\mathcal{J}_{1}^{N_{p}}$ is the future discrete time window. $y_{\text {ref }}$ contains the description of reference behavior, $\tilde{u}$ is the sought sequence of the future MV $u$ of the process that is classically tuned as follow:

$$
\tilde{u}=[\ldots u(j) \ldots]^{T} \quad \forall j \in \mathcal{J}_{0}^{N_{c}-1}=\left\{k, \ldots, k+N_{c}-1\right\}
$$

where $N_{c}$ is the control horizon and where:

$$
u(j)=u\left(k+N_{c}-1\right) \forall j \in \mathcal{J}_{N_{c}}^{N_{p}-1}=\left\{k+N_{c}, \ldots, k+N_{p}-1\right\}
$$

This optimization problem has also to account for discrete time versions of the continuous time version constraints (2) and (3): 
- constraints on the magnitude and velocity of the MV:

$$
\left\{\begin{array}{l}
u_{\text {min }} \leq u(j) \leq u_{\max } \quad \forall j \in \mathcal{J}_{0}^{N_{p}-1}=\left\{k, \ldots, k+N_{p}-1\right\} \\
\Delta u_{\min } \leq u(j)-u(j-1) \leq \Delta u_{\max } \quad \forall j \in \mathcal{J}_{0}^{N_{p}-1}
\end{array}\right.
$$

- $n$ general output constraints for the CV:

$$
c_{i}\left(y_{r e f}(j), y_{p}(j), u(j-1)\right) \leq 0 \quad \forall j \in \mathcal{J}_{1}^{N_{p}}, \forall i \in \mathcal{I}_{1}^{n}
$$

In the performance index given in (6) and in the output constraints formulated in (10), one needs, at the current discrete time $k$, the value of the future measurements $y_{p}$ over the prediction horizon $N_{p}$. This impossibility can be handled using the internal model control (IMC) structure [20,29] with MPC as depicted Fig. 1. In this structure, the MV is applied to both the process and the model.

In this control structure combined in the MPC structure, the error $e$ is:

$$
e(j)=y_{p}(j)-y_{m}(j)
$$

In our approach, the difference $e$ between process and model $\mathrm{CV}$ is fed back into the controller, but also is the model CV. Two feedback loops are therefore used in this IMC-MPC structure to adjust process performances: not only using the classical feedback error but also with the correction of modeling errors introduced in the model-based on-line optimizer.

Assumption 4 [18,20] At each sample time $k$, the error $e(j)$ between the process output and the model output remains the same over the prediction horizon $N_{p}$. The error value is updated at each time $k$.

This assumption and Eq. (11) allow to introduce the model $\left(\mathcal{S}_{N L}\right)$ and the 
feedback term $e(k)$ into the constrained optimization problem:

$$
\left\{\begin{array}{l}
\min _{\tilde{u}} J(\tilde{u})=\sum_{j \in \mathcal{J}_{1}^{N_{p}}} g\left(y_{r e f}(j), y_{m}(j), u(j-1), e(k)\right) \\
\tilde{u}=[\ldots u(j) \ldots]^{T} \quad \forall j \in \mathcal{J}_{0}^{N_{c}-1} \\
u(j)=u\left(k+N_{c}-1\right) \quad \forall j \in \mathcal{J}_{N_{c}}^{N_{p}-1} \\
u_{\text {min }} \leq u(j) \leq u_{\max } \quad \forall j \in \mathcal{J}_{0}^{N_{p}-1} \\
\Delta u_{\text {min }} \leq u(j)-u(j-1) \leq \Delta u_{\text {max }} \quad \forall j \in \mathcal{J}_{0}^{N_{p}-1} \\
c_{i}\left(y_{\text {ref }}(j), y_{m}(j), u(j-1), e(k)\right) \leq 0 \quad \forall j \in \mathcal{J}_{1}^{N_{p}}, \forall i \in \mathcal{I}_{1}^{n} \\
\text { and subject to the resolution of the model }\left(\mathcal{S}_{N L}\right) .
\end{array}\right.
$$

Remark 2.1 $y_{m}(j)$ is the sampled value obtained from the resolution of the continuous model $\left(\mathcal{S}_{N L}\right)$.

\subsection{Off-line and on-line IMC-MPC structures}

On-line computational time is dealing with the resolution of the optimization problem that includes the model resolution. Discretization of the PDE model can lead to a large amount of algebraic differential equations (DAES) that increases the computational burden, especially in the nonlinear case. In order to reduce the on-line computational time, the IMC-MPC structure is used offline for the system $\left(\mathcal{S}_{N L}\right)$. As previously described, given small input variation $\Delta u$, small state variations $\Delta \underline{x}$ and small output variation $\Delta y_{m}$ about $\left(S_{0}\right)$ can be represented through the time-varying linearized model $\left(\mathcal{S}_{T V L}\right)$. Finally, 
the off-line solved nonlinear model $\left(S_{0}\right)$ and the on-line solved time-varying linearized model $\left(S_{T V L}\right)$ replace the initial nonlinear model $\left(\mathcal{S}_{N L}\right)$ in the IMCMPC structure as depicted Fig. 2.

The control objective is now to find the variation $\Delta u$ of the manipulated variable $u$ about a chosen trajectory $u_{0}$ leading to a better on-line optimization result. According to the time-varying linearized IMC structure, the control problem is now equivalent to the following constrained optimization problem:

$$
\left\{\begin{array}{l}
\min _{\tilde{u}} J(\tilde{u})=\sum_{j \in \mathcal{J}_{1}^{N_{p}}} g\left(y_{\text {ref }}(j), \Delta y_{m}(j), \Delta u(j-1), e(k)\right) \\
\Delta \tilde{u}=[\ldots \Delta u(j) \ldots]^{T} \quad \forall j \in \mathcal{J}_{0}^{N_{c}-1} \\
\Delta u(j)=\Delta u\left(k+N_{c}-1\right) \quad \forall j \in \mathcal{J}_{N_{c}}^{N_{p}-1} \\
u_{\text {min }}-u_{0}(j) \leq \Delta u(j) \leq u_{\max }-u_{0}(j) \quad \forall j \in \mathcal{J}_{0}^{N_{p}-1} \\
\Delta u_{\text {min }}^{\prime} \leq \Delta u(j)-\Delta u(j-1) \leq \Delta u_{\text {max }}^{\prime} \quad \forall j \in \mathcal{J}_{0}^{N_{p}-1} \\
\Delta u_{\min }^{\prime}=\Delta u_{\min }-\left(u_{0}(j)-u_{0}(j-1)\right) \quad \forall j \in \mathcal{J}_{0}^{N_{p}-1} \\
\Delta u_{\text {max }}^{\prime}=\Delta u_{\max }-\left(u_{0}(j)-u_{0}(j-1)\right) \quad \forall j \in \mathcal{J}_{0}^{N_{p}-1} \\
c_{i}\left(y_{\text {ref }}(j), \Delta y_{m}(j), \Delta u(j-1), e(k)\right) \leq 0 \quad \forall j \in \mathcal{J}_{1}^{N_{p}}, \quad \forall i \in \mathcal{I}_{1}^{n} \\
\text { and subject to the resolution of the model }\left(\mathcal{S}_{T V L}\right) .
\end{array}\right.
$$

The next step now developed is concerned with methods to handle constraints during the on-line optimization problem resolution. 


\subsection{Constraints handling}

Two different kind of constraints are to be accounted for: constraints acting only on MV and constraints acting on CV (and eventually on MV also).

\subsubsection{Input constraints handling}

Transformation method for variables allows to translate explicit constraints on the optimization argument $u$ (and only the optimization argument) as new equations for new unconstrained argument $p$ [13]. Here, we propose to enlarge this method to cover magnitude constraints and velocity constraints as well (acceleration rate constraints may also be accounted for). In order to be used in the optimization algorithm, such a transformation must have a first derivative different from zero and must be invertible. This leads to a transformation equation (Fig. 3) like the transformation used in [25]:

where:

$$
\left\{\begin{array}{l}
u(j)=f(p(j))=f_{\text {moy }}+f_{\text {amp }} \tanh \left(\frac{p(j)-f_{\text {moy }}}{f_{\text {amp }}}\right) \quad \forall j \in \mathcal{J}_{0}^{N_{c}-1} \\
p(j) \in \mathbb{R} \quad \forall j \in \mathcal{J}_{0}^{N_{c}-1}
\end{array}\right.
$$

with the time-varying coefficients $f_{\text {moy }}$ and $f_{\text {amp }}$ updated at each time $k$ : 


$$
\begin{cases}f_{\text {moy }}=\frac{f_{\max }+f_{\min }}{2} & \\ f_{\text {amp }}=\frac{f_{\max }-f_{\min }}{2} & \\ f_{\min }=\max \left(u_{\min }, u(j-1)+\Delta u_{\min }\right) & \forall j \in \mathcal{J}_{0}^{N_{c}-1} \\ f_{\text {max }}=\min \left(u_{\max }, u(j-1)+\Delta u_{\text {max }}\right) & \forall j \in \mathcal{J}_{0}^{N_{c}-1}\end{cases}
$$

Moreover, such time-varying coefficients $f_{\max }$ et $f_{\min }$ ensure that the first derivative is equal to one is the middle of the constrained range. Therefore, in this zone, the optimization procedure is not disturbed by the sensitivity of the transformation, which is not the case near the constraints bound.

\subsubsection{Output constraints handling}

In order to take account for output constraints as soft constraints (for which violation may be allowed [26]), we adopt the exterior penalty method [13] used in nonlinear programming where a positive defined weighted penalty term is added to the initial cost function $J$ :

$$
\left\{\begin{array}{l}
J_{t o t}=J+J_{e x t} \\
J_{e x t}=\sum_{j \in \mathcal{J}_{1}^{N p}}\left(\sum_{i \in \mathcal{I}_{1}^{n}} w_{i} \max ^{2}\left(0, c_{i}\left(y_{r e f}(j), \Delta y_{m}(j), \Delta u(j-1), e(k)\right)\right)\right)^{(16)}
\end{array}\right.
$$

where $w_{i}$ is an adaptive positive defined weight: it increases when the related constraint tends to be checked, otherwise it decreases. Roughly speaking, for any constraint $c_{i}$ not checked (i.e when $c_{i}\left(y_{r e f}(j), \Delta y_{m}(j), \Delta u(j-1), e(k)\right)>$ 0 ), a positive term penalize the minimization task. This enforces the optimizer to minimize $J_{e x t}$ and hence to enforce the violated constraint to be checked. 
The penalty method transforms the problem into an unconstrained problem by substituting a penalty function for the constraint. Therefore, the constrained solution might be approached from the unfeasible side of the constraints: this relative drawback can be an advantage in the case where, sometimes, no solution for the constrained problem exists [10] and constraint violation has therefore to be allowed.

\subsection{Final penalized optimization problem}

Finally, combining the transformation method for the constraints on the MV and the exterior penalty method for the constraints on the $\mathrm{CV}$, the final penalized optimization problem to be solved on-line is the following one:

$$
\left\{\begin{array}{l}
\min _{\Delta \tilde{p}} J_{t o t}(\Delta \tilde{p})=\sum_{j \in \mathcal{J}_{1}^{N_{p}}} g\left(y_{r e f}(j), \Delta y_{m}(j), \Delta p(j-1), e(k)\right)+ \\
\qquad \sum_{j \in \mathcal{J}_{1}^{N_{p}}}\left(\sum_{i \in \mathcal{I}_{1}^{n}} w_{i} \max ^{2}\left(0, c_{i}\left(y_{\text {ref }}(j), \Delta y_{m}(j), \Delta p(j-1), e(k)\right)\right)\right. \\
\Delta \tilde{p}=\left[\ldots f^{-1}(\Delta u(j)) \ldots\right]^{T} \forall j \in \mathcal{J}_{0}^{N_{c}-1} \\
\Delta p(j)=\Delta p\left(k+N_{c}-1\right) \forall j \in \mathcal{J}_{N_{c}}^{N_{p}-1} \\
\Delta u(j)=u(j)-u_{0}(j)=f\left(p_{0}(j)+\Delta p(j)\right)-f\left(p_{0}(j)\right) \forall j \in \mathcal{J}_{0}^{N_{c}-1} \\
p_{0}(j)=f^{-1}\left(u_{0}(j)\right) \forall j \in \mathcal{J}_{0}^{N_{c}-1} \\
\text { and subject to the resolution of the model }\left(\mathcal{S}_{T V L}\right) .
\end{array}\right.
$$

This penalized problem can now be solved by any unconstrained optimization algorithm. 


\subsection{Control algorithm}

Widely known for its robustness and convergence properties, we use of the well-known Levenberg-Marquardt's algorithm, where the argument $\Delta \tilde{p}$ is determined at each sample instant $k$ by the iteration procedure:

$$
\Delta \tilde{p}^{n+1}=\Delta \tilde{p}^{n}-\left(\nabla^{2} J_{t o t}^{n}+\lambda I\right)^{-1} \nabla J_{t o t}^{n}
$$

where $\nabla J_{t o t}^{n}$ and $\nabla^{2} J_{t o t}^{n}$ are the criteria gradient and the criteria hessian with respect to $\Delta \tilde{p}^{n}$ at the iteration $n \cdot \nabla J_{\text {tot }}^{n}$ is explicitly provided into the control algorithm and the classical Gauss's approximation for $\nabla^{2} J_{t o t}^{n}$ as well. This IMC-MPC resolution algorithm is now implemented for control of the drying process and experimental results are given in next section.

\section{Experimental control of a drying process}

The aim of this section is to evaluate the IMC-MPC formulation for control of a drying process. The drying procedure is first described and the control problem is introduced. Then, experimental results to illustrate the proposed IMC-MPC structure are shown and discussed.

\subsection{Introduction}

Reactive painting drying is an important industrial problem through its impact for the quality of the final aspect of products in many industries (cars, preglass plaint sheets, cans, etc.). The main difficulty encountered during this operation is the evacuation of the solvent before the end of polymerization 
reaction: this solvent is needed to simultaneously build the painting film and to ensure the film polymerization but one should avoid to trap it in the final dried product. Until now, the use of volatile organic compounds (VOCs) allowed the control of this evacuation during the polymerization reactions. But the enforced regulations concerning environment lead now to replace the use of VOCs by the use of water based solvent. However, compared with VOCs, they evacuate less rapidly and at higher temperatures. To overcome this problem, infrared drying has been widely developed in industrial processes. Some studies $[22,28,30]$ deal with diffusion problems with both infrared drying and water used as solvent.

Within the framework of a previous study [6], an experimental drying process has been built. It allows to dry a painting film sample coated on a car iron support by supply of an infrared flow. The experimental plant is represented Fig. 4 with the infrared part and with the instrumentation part. The sensors are: a pyrometer that allows the on-line temperature measurement of the sample at the upper surface and a precision balance that allows the follow-up of the sample and support set mass. The painting film sample mass is the sum of the constant final dried mass of the sample with the time varying water mass in the sample. Indeed, during the drying under infrared flow, this water mass decreases since the water contained in the painting film sample migrates from inside to the upper surface and vaporizes at this surface.

\subsection{Control objective}

In term of final quality of the film, painting formulation (and particularly the solvent) does not alone lead to cope with the delicate balance problem 
between water extraction as fast as possible and polymerization reaction to get a acceptable final quality. Indeed, for real applications, the final product obtained by the painting film sample drying has to be usable: bubbles and fissures phenomena have therefore to be avoided. To ensure the final product quality, paint producers propose to follow a reference temperature trajectory during the drying cycle (temperature rising at constant velocity and upholding at a given temperature). The control problem considered here is the tracking of a reference trajectory $y_{r e f}$ for the process temperature $(\mathrm{CV})$, subject to constraints on the infrared flow (MV).

\section{$3.3 \quad$ Modeling}

During the overall drying procedure, two phenomena are taking place: first drying of the painting film and then polymerization reaction. Since polymerization reaction dynamics is not important when the drying takes place, only drying phenomena is accounted for in the model. Mass and temperature measurements available during experimental drying kinetics have allowed to validate the first principles model that is used in this paper [6]: the low thickness painting film sample is characterized by its temperature assumed uniform $T(t)$ and by its dry basis humidity $\chi(z, t)$ assumed to varying only according to the thickness $z$ of the sample. The drying leads to water losses which produces a variation in the sample geometry. Considering the low thickness of the sample with respect to the large surface size, one consider that the water extraction leads only to the linear reduction of the sample thickness $e_{p}$ with respect to the mean humidity in dry basis $\bar{\chi}$ :

$$
e_{p}=e_{s e c}(1+\phi \bar{\chi})
$$


where $e_{\text {sec }}$ is the final constant dried thickness of the sample and with :

$$
\bar{\chi}(t)=\frac{1}{e_{s e c}} \int_{0}^{e_{s e c}} \chi(z, t) d z \forall t>0
$$

The model of the painting film sample infrared drying can finally be repre-

sented by the state variables $T(t)$ and $\chi(z, t)$. It is deduced from the following energy and mass balances (remaining expressions and coefficients value are given in [17]).

\subsubsection{Energy balance}

One assume that the car iron support is a sufficiently good thermal conductor to consider that the temperature $T$ is uniform on the sample and support set: $T$ is therefore only time dependent. Taking into account of the different losses $P_{i}$ as well as the absorbed infrared flow represented Fig. 5,

the energy balance leads to, $\forall t>0, \forall z \in\left[0, e_{s e c}\right]$ :

$$
\begin{aligned}
\left(\rho_{p} C_{p}(\bar{\chi}, T) e_{p}+\rho_{s} C_{s} e_{s}\right) \frac{d T}{d t}= & h_{c}\left(T_{h}-T\right)+\sigma_{h}\left(T_{h}^{4}-T^{4}\right) \\
& +h_{c}\left(T_{b}-T\right)+\alpha_{s} \sigma\left(T_{b}^{4}-T^{4}\right) \\
& -l_{v}(T) \dot{m}(\bar{\chi}, T)+\alpha_{i r}(\bar{\chi}) \varphi_{i r}
\end{aligned}
$$

where $\rho_{p} C_{p}(\bar{\chi}, T) e_{p}$ and $\rho_{s} C_{s} e_{s}$ are respectively the surface thermal capacity of the painting film sample and the surface thermal capacity of the support. The four first terms on the right-hand side of Eq. (21) are natural convection and radiation phenomena on both upper and lower surfaces. The fifth term on the right-hand side of Eq. (21) deals with the loss of water due to evaporation at upper surface. The MV is the infrared flow $\varphi_{i r}(t)$ and acts the boundary $z=e_{\text {sec }}$. 
Remark 1 According to the spatial uniform property assumption on the temperature, the temperature evolution is described by an nonlinear ordinary differentialintegral equation.

\subsubsection{Mass balance}

Since there is no macro-porous structure, we consider that the water migrates only by diffusion phenomenon. It allows to write the mass balance using the Fick's law:

- $\forall t>0, z \in \Omega=] 0, e_{s e c}[$ :

$$
\frac{\partial \chi}{\partial t}=\frac{\partial}{\partial z}\left(D_{e f f}(\chi, T) \frac{\partial \chi}{\partial z}\right)
$$

with the effective diffusion coefficient $D_{\text {eff }}$ depending on the humidity, the temperature and the linear reduction of the thickness:

$$
D_{e f f}(\chi, T)=\frac{D_{0} \exp \left(\frac{-a}{\chi}\right)}{(1+\phi \chi)^{2}}
$$

- $\forall t>0$, at $z=0$ (i.e. at the painting film sample lower surface), there is no mater transfer:

$$
\frac{\partial \chi}{\partial z}=0
$$

- $\forall t>0$, at $z=e_{\text {sec }}$ (i.e. at the painting film sample upper surface), the outgoing flow is linked to the drying velocity $\dot{m}$ through:

$$
-D_{e f f}(\chi, T) \frac{\partial \chi}{\partial z}=\frac{\dot{m}(\bar{\chi}, T)}{\rho_{p}}
$$

with the drying velocity given by [5]:

$$
\dot{m}(\bar{\chi}, T)=\frac{k_{m} m_{v}}{R} P_{t} \frac{2}{T+T_{h}} \log _{10}\left[\frac{P_{t}-\chi_{a i r} P_{v_{s a t}}\left(T_{h}\right)}{P_{t}-a_{w}(\bar{\chi}) P_{v_{s a t}}(T)}\right]
$$


The reader is referred to [17] for more details about the expressions of $P_{v_{s a t}}(T)$ and $a_{w}(\bar{\chi})$

Finally, the model is composed of two coupled equations : a nonlinear ordinary differential-integral equation and a nonlinear parabolic PDE.

\subsection{Model Predictive Control formulation}

In the MPC framework previously described, the mathematical discrete-time formulation is written here as the following constrained optimization problem:

$$
\min _{\tilde{u}} J(\tilde{u})=\sum_{j \in \mathcal{J}_{1}^{N p}}\left(y_{r e f}(j)-y_{p}(j)\right)^{2}
$$

with magnitude and velocity constraints for the MV.

\subsection{Experimental results}

In this first attempt, the control horizon $N_{c}$ is tuned to 1 : even if it tends to give less interesting solution, it allows to minimize the on-line computational time. Experiments have been realized to point out the prediction horizon influence over the tracking performance.

\subsubsection{Operating conditions}

The operating conditions are the following one:

- the linearization about $\left(\mathcal{S}_{0}\right)$ is performed with $u_{0}=5000 \mathrm{~W} \cdot \mathrm{m}^{-2}$ and with the initial conditions $T_{i}=36{ }^{\circ} \mathrm{C}$ and $\chi_{i}=0.4 \mathrm{~kg}_{\mathrm{kg}} \mathrm{kg}^{-1}$ 
- the models $\left(\mathcal{S}_{0}\right)$ and $\left(S_{T V L}\right)$ are solved by the finite volumes method $(6$ volumes) using the subroutine ddaspg from IMSL library ;

- the value for the sampling period $T_{e}$ is $1 s$;

- constraints boundaries are:

$$
\begin{aligned}
u_{\max } & =12,000 W \cdot m^{-2} \\
u_{\min } & =0 W \cdot m^{-2} \\
\Delta u_{\max } & =+500 W \cdot m^{-2} \\
\Delta u_{\min } & =-500 W \cdot m^{-2}
\end{aligned}
$$

- atmospheric conditions are:

$$
\begin{aligned}
\chi_{a i r} & =20 \% \\
T_{h} & =52{ }^{\circ} \mathrm{C} \\
T_{b} & =20{ }^{\circ} \mathrm{C}
\end{aligned}
$$

- the control algorithm, written in Fortran code, has been combined to C code in order to realize the interface with the sensor and the actuator ;

- the processor rate is $400 \mathrm{MHz}$.

In order to compare results for any value of $N_{p}$, one introduces the normalized cost function $J_{t o t}^{\prime}=\frac{J_{t o t}}{N_{p}}$.

\subsubsection{Temperature reference trajectory tracking}

From the reference tracking and the tracking error results (resp. depicted Fig. 6 and Fig. 7), we can see that the tracking objective is correctly achieved.

Moreover, the intermediate value 6 for the horizon prediction gives the best result:

- with a small prediction horizon $\left(N_{p}=3\right)$, the handling of the slope variation of the reference signal (around $k=80,120$ and 200) is less efficient than 
with $N_{p}=6$ as one can see for the values taken by the normalized cost function (Fig. 8). In this case, informations quantity available describing the future process behavior is insufficient. In a way, with $N_{p}=3$ the problem is badly stated for its resolution, as we can see on the applied control: when the three slope variations appear, the infrared flow is always either saturated in magnitude (Fig. 9) or in velocity (Fig. 10). The optimizer does not correctly capture the future behavior of the process. Then, the algorithm tends too often to find a non admissible solution and to do bang-bang control. This leads consequently to poor tracking performances ;

- increasing the prediction horizon value to 6 and 12, the infrared flow becomes smoother (Fig. 9), but with a large prediction horizon $\left(N_{p}=12\right)$, another problem appears for $0 \leq k \leq 80$ : in term of variation, the model output, which trend is similar to the process output, is quantitatively too different from the process output (Fig. 11). This is a limitation due to modeling errors and therefore to related linearization errors. Since more values calculated by the model resolution are taken into account in the optimization problem, the criteria minimization is less efficient than in the case where the prediction horizon take an average value for $\left(N_{p}=6\right)$ as clearly depicted for the normalized cost function (Fig. 8). This is confirm when tuning $N_{p}$ to values higher than 12 .

Finally, the prediction horizon value $N_{p}=6$ is the optimal choice for this main parameter: experiments with $N_{p}$ tuned to $5,7,8,9,10,11$ or more were giving less interesting results. Moreover, one of the property of the IMC structure (integral action), and the interest of this IMC-MPC strategy, is confirmed by these experimental results: the tracking is effective in spite of the model output used in the control algorithm does not track quantitatively the 
temperature reference trajectory (Fig. 11). Concerning the hyperbolic transformation, relative lost of sensitivity near the constraints bound does not affect overall performance during the real-time application.

\section{Conclusion}

This paper is dealing with the control of systems described by nonlinear parabolic partial differential equations. The idea is to developed a general MPC framework combined with IMC structure that can be used for a large amount of practical applications. Two feedback loops are used in this IMCMPC structure in order to correct process performances and modeling errors introduced in the model-based on-line optimizer. IMC structure allows to use less accurate and therefore less time consuming resolution method for the optimization problem. Moreover, in order to decrease the on-line computational time needed to solve the final penalized optimization problem, a linearization method of the model is used. Input constraints due to natural actuators limitations are handled through a transformation law that allows to always check these constraints. Soft constraints, for which violation may be allowed, are handled through an exterior penalty method. This control strategy is applied for a painting film drying process where a trajectory tracking under input constraints is stated. Interesting performance are shown for experiments and the influence of the MPC tuning parameter, i.e. the horizon prediction, is pointed out. Is also allows to see the relative robustness property of the IMC-MPC structure with respect to modeling errors. 


\section{References}

[1] Allgöwer, F., Badgwell, T.A., Qin, J.S., Rawlings, J.B., \& Wright, S.J. (1999). Nonlinear predictive control and moving horizon estimation. In P.M. Frank (Ed.) Advances in control. Highlights of ECC'99, Springer, Berlin.

[2] Armaou, A., \& Christofides, P.D. (2002). Dynamic optimization of dissipative PDE systems using nonlinear order reduction. Chem. Eng. Sci., 57(24), 50835114.

[3] Balas, M.J. (1998). Stable feedback control of linear distributed parameter systems: time and frequency domain conditions. J. Math. Anal. Appl., 225(1), 144-167.

[4] Barbu, V. (1993). Analysis and control of nonlinear infinite dimensional systems. Academic Press Inc.

[5] Bird, R.B., Stewart, W.E., \& Lighfoot, E.N. (1960). Transport phenomena. John Wiley and Sons.

[6] Blanc, D., Laurent, P., \& Andrieu, J. (1997). Modeling of the reactive infrared drying of a model water-based epoxy-amine painting coated on iron support with experimental validation. Proc. of the 11th IHTC, 5, 181-186.

[7] Christofides, P.D., \& Daoutidis, P. (1997). Finite-dimensional control of parabolic PDE systems using approximate inertial manifolds. J. Math. Anal. Appl., 216(2), 398-420.

[8] Christofides, P.D. (1998). Robust control of parabolic PDE systems. Chem. Eng. Sci., 53(16), 2949-2965.

[9] Christofides, P.D. (2001). Nonlinear and robust control of PDE systems: methods and application to transport-reaction processes. Birkhäuser, Boston. 
[10] Dufour, P., Couenne, F., \& Touré, Y. (accepted). Model predictive control of a catalytic reverse flow reactor", special issue of IEEE Trans. on Control Syst. Technol. on Control of Industrial Spatially Distributed Parameter Processes.

[11] Eder, H.H. (1999). MBPC Benefits and key success factors. Proc. of the $E C C$, Paper F1033-6.

[12] El-Farra, N.H., Armaou, A., \& Christofides, P.D. (accepted). Analysis and control of parabolic PDE systems with input constraints. Automatica.

[13] Fletcher, R. (1987). Practical methods of optimization. John Wiley and Sons.

[14] Friedly, J.C. (1972). Dynamic behavior of processes. Prentice-Hall, Inc.

[15] Godasi, S., Karakas, A., \& Palazoglu, A. (2002). Control of nonlinear distributed parameter processes using symmetry groups and invariance conditions. Comput. G Chem. Eng., 26(7-8), 1023-1036.

[16] Hoo, K.A., \& Zheng, D. (2001). Low-order control-relevant models for a class of distributed parameter systems. Chem. Eng. Sci., 56(23), 6683-6710.

[17] Larabi, M.C., Dufour, P., Laurent, P., \& Touré, Y. (2000). Predictive control of a nonlinear distributed parameter system: real time control of a painting film drying process. Proc. of the MTNS conference, Paper B167.

[18] Marquis, P., \& Broustail, J.P. (1988). SMOC, a bridge between state space and model predictive controllers: application to the automation of a hydrotreating unit. Proc. of the IFAC Workshop on Model Based Process Control, 37-43.

[19] Mayne, D.Q., Rawlings, J.B., Rao, C.V., \& Scokaert, P.O.M. (2000). Constrained model predictive control: stability and optimality. Automatica, $36(6), 789-814$.

[20] Morari, M., \& Zafiriou, E. (1989). Robust process control. Dunod. 
[21] Morari, M., \& Lee, J.H. (1999). Model predictive control: past, present and future. Comput. \& Chem. Eng., 23(4-5), 667-682.

[22] Okazaki, M., Shioda, K., Masuda, K., \& Toei, R. (1974). Drying mechanism of coated film of polymer solution. J. of Chem. Eng. of Jpn., 7(2), 99-105.

[23] Palazolu, A., \& Karakas, A. (2000). Control of nonlinear distributed parameter systems using generalized invariants. Automatica, 36(5), 697-703.

[24] Pazy, A. (1983). Semigroups of linear operators and applications to partial differential equations. Springer Verlag New-York.

[25] Polak, E. (1971). Computational Methods in Optimization. Academic Press.

[26] Qin, J.S, \& Badgwell, T.A. (in press). A survey of industrial model predictive control technology. Control Eng. Pract.

[27] Rawlings, J.B (2000). Tutorial overview of model predictive control. IEEE Control Syst., 20(3), 38-52.

[28] Sano, Y. (1992). Drying of Polymer Solution. Drying Technol., 10(3), 591-622.

[29] Touré, Y., \& Josserand, L. (1997). An extension of IMC to boundary control of distributed parameter systems. Proc. of the IEEE International Conference on Systems, Man and Cybernetics-CCS, Orlando, USA, 3, 2426-2431,.

[30] Vrentas, J.S., \& Vrentas, C.M. (1994). Drying of solvent-coated polymer films. J. Polym. Sci., Part B: Polym. Phys., 32, 187-194.

[31] Zheng, D., \& Hoo, K.A. (2002). Low-order model identification for implementable control solutions of distributed parameter systems. Comput. $\mathscr{G}$ Chem. Eng., 26(7-8), 1049-1076. 


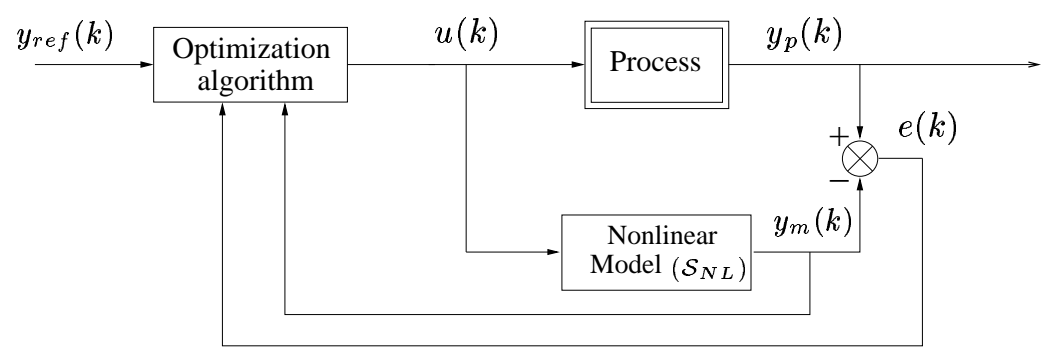

Fig. 1. General IMC-MPC structure.

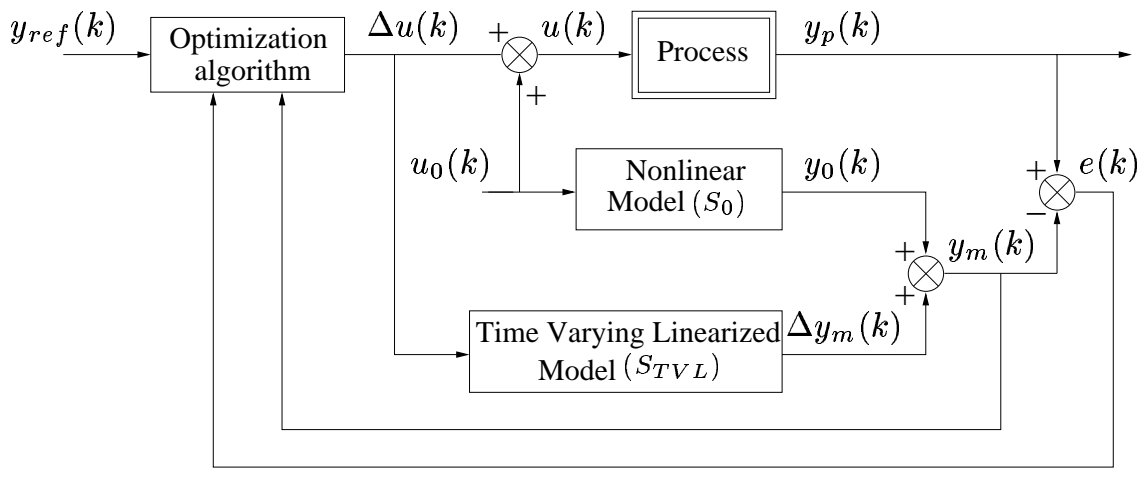

Fig. 2. General Linearized IMC-MPC structure.

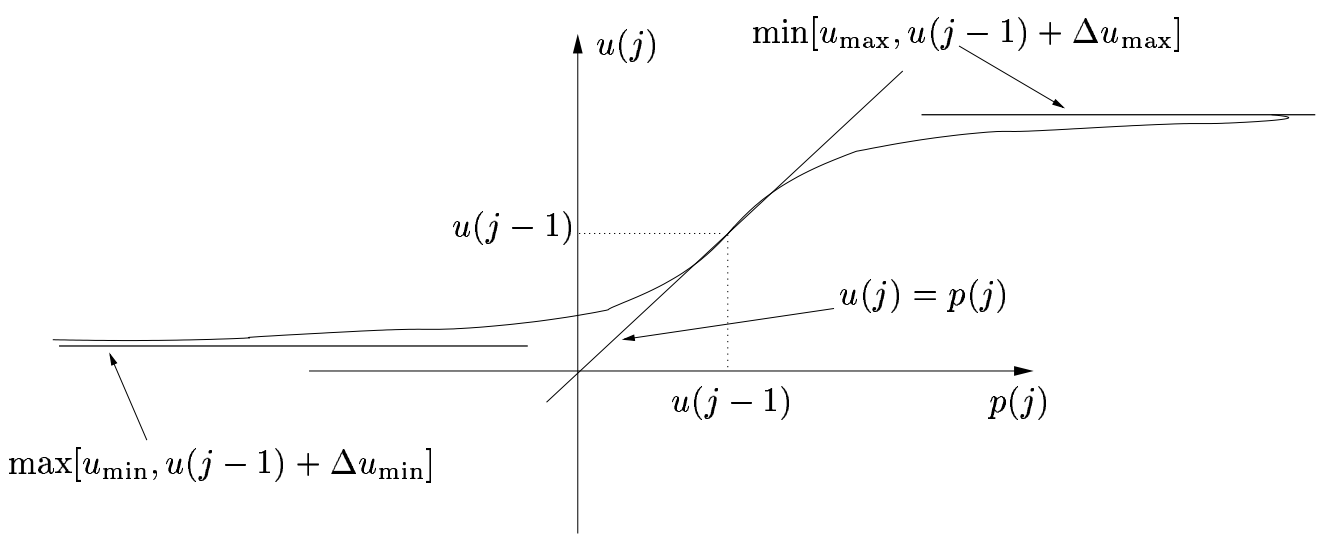

Fig. 3. Mapping from unconstrained variable $p$ into constrained variable $u$. 


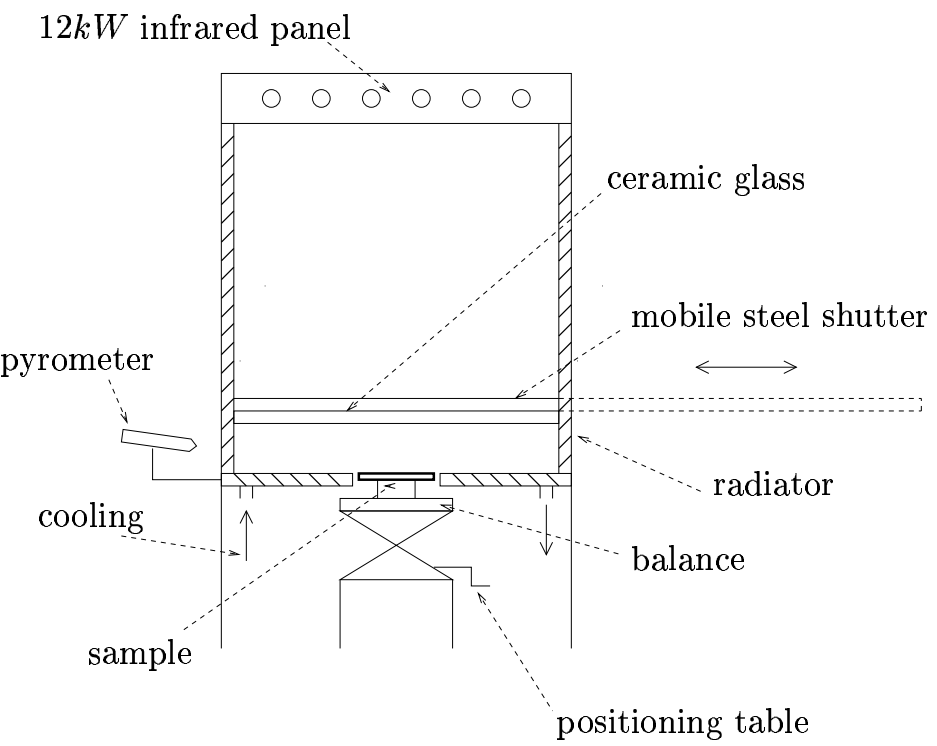

Fig. 4. Drying process.

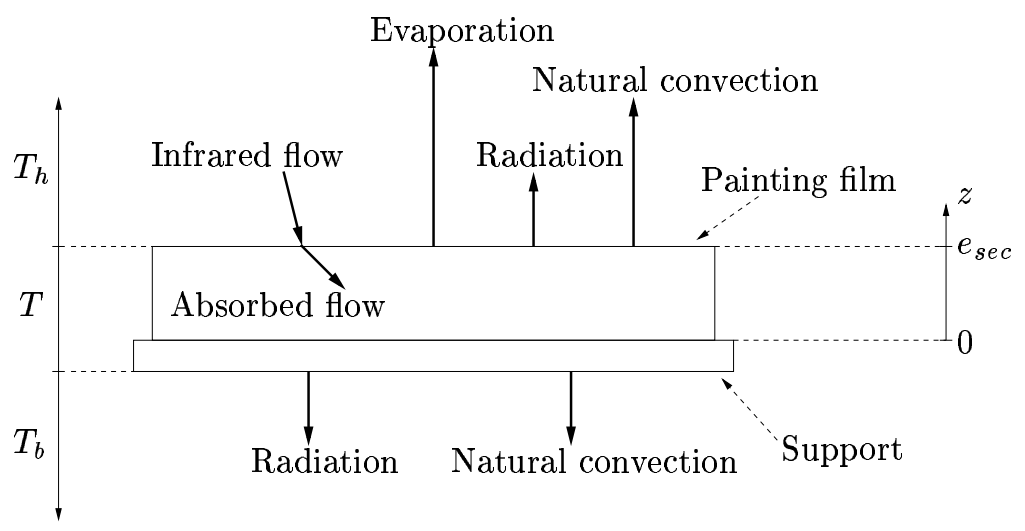

Fig. 5. Thermal flows occurring during the drying procedure. 


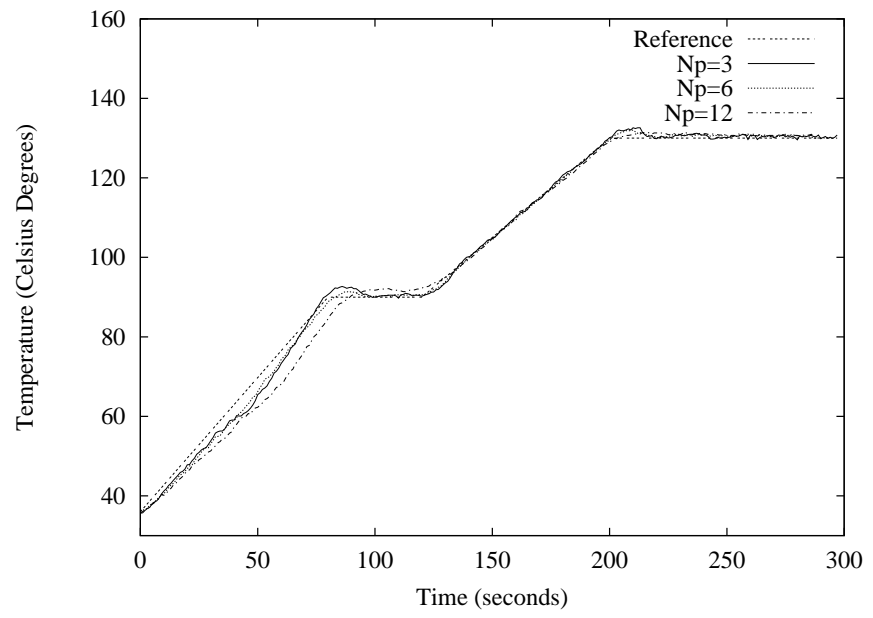

Fig. 6. Reference tracking for $N_{p}=3,6,12$.

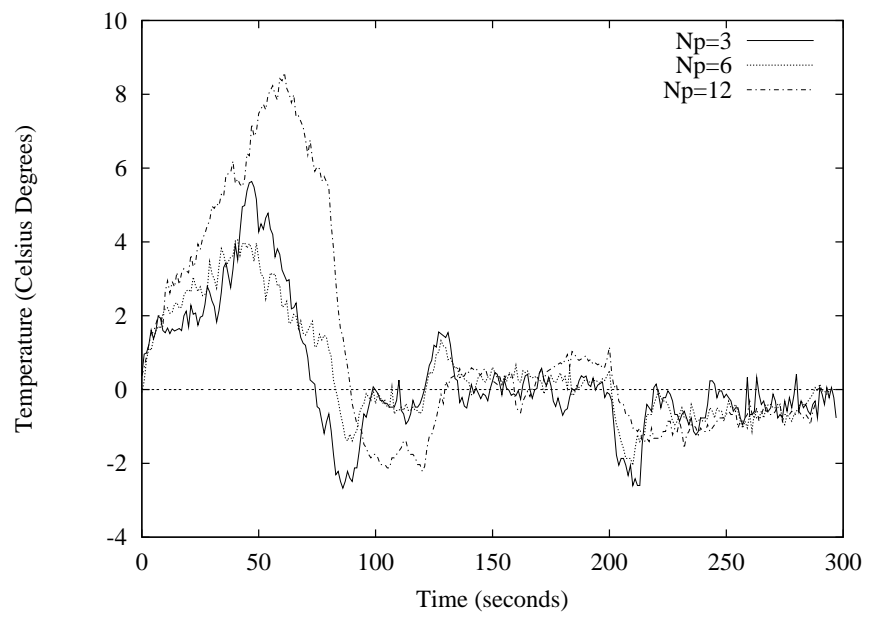

Fig. 7. Tracking error for $N_{p}=3,6,12$. 


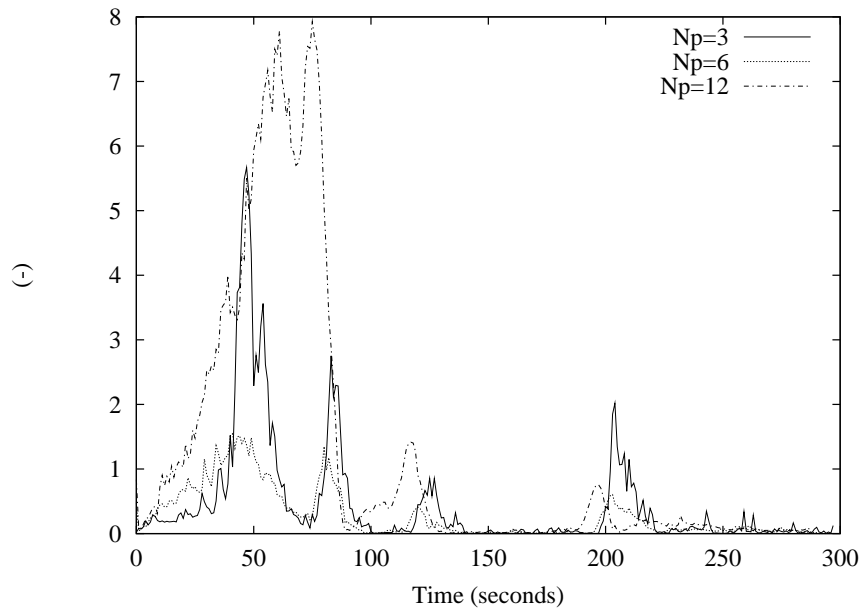

Fig. 8. Normalized criteria value sequence for $N_{p}=3,6,12$.

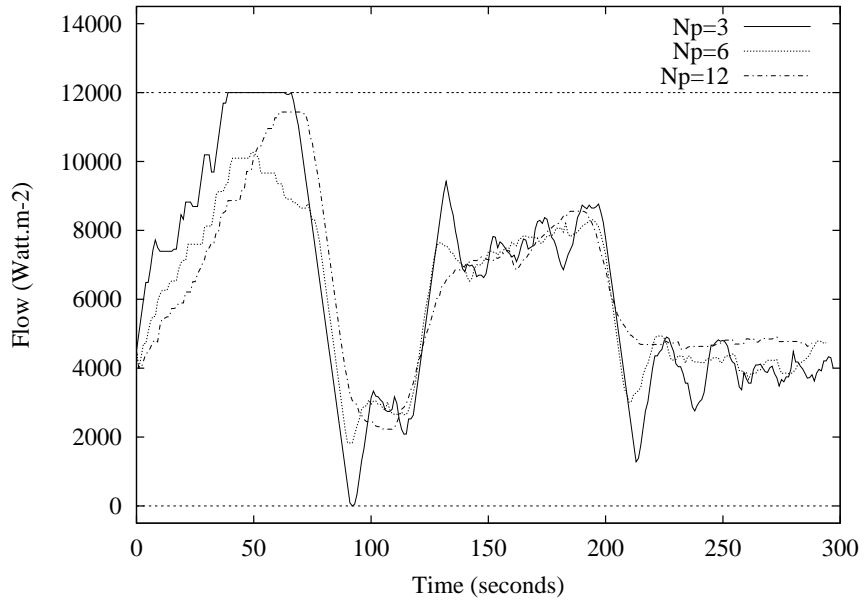

Fig. 9. Control magnitude for $N_{p}=3,6,12$. 


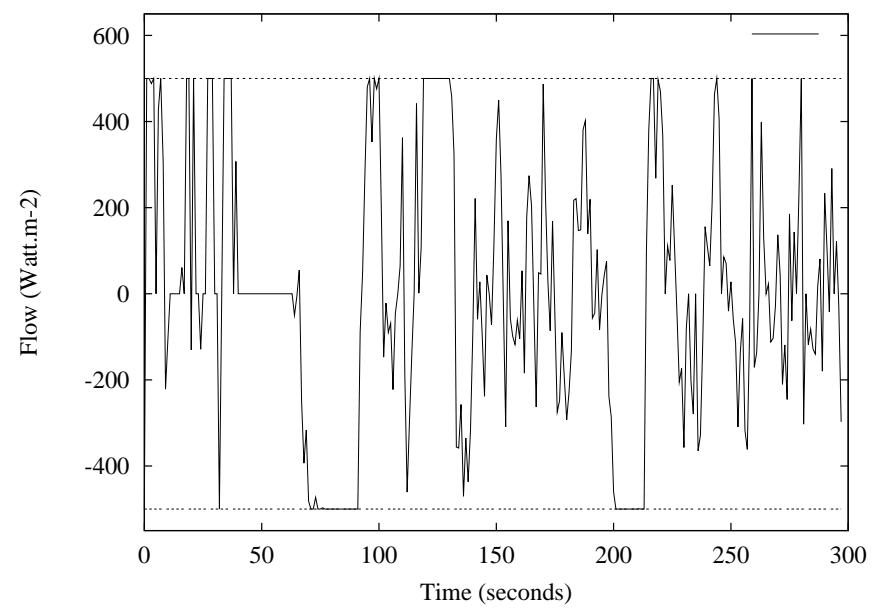

Fig. 10. Control variation for $N_{p}=3$.

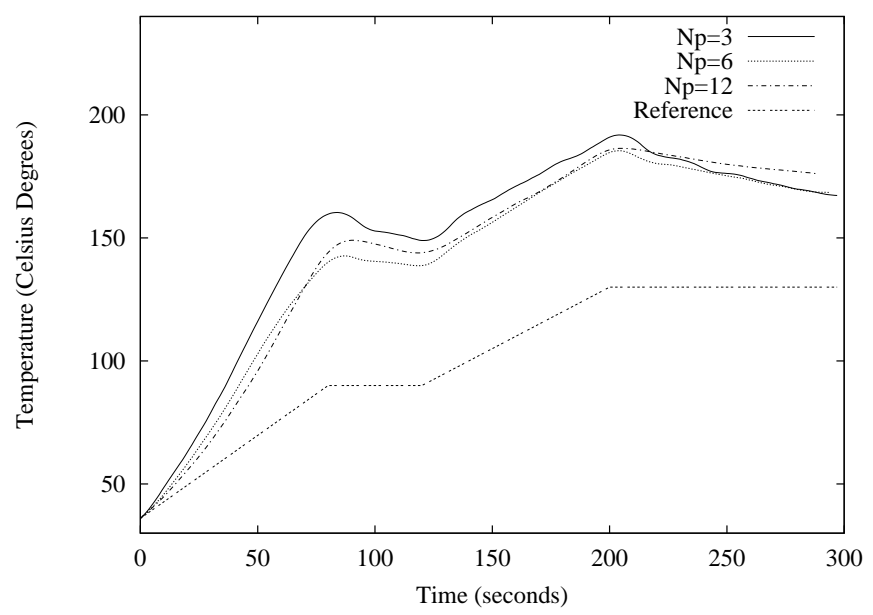

Fig. 11. Model output for $N_{p}=3,6,12$. 